\title{
Automatic Scheme for Inelastic Column Buckling
}

\author{
Raghavan Ramalingam, S. Arul Jayachandran \\ Department of Civil Engineering \\ Indian Institute of Technology Madras, Chennai, India \\ raghavan.ramalingam@gmail.com; aruls@iitm.ac.in
}

\begin{abstract}
The inelastic buckling of columns is difficult to model analytically. Methods like FEM or approximate closed form expressions are presently available but they are unsatisfactory for reasons mentioned in this paper. In this study, the inelastic buckling of columns is modelled to reflect the true curves upto the ultimate load prior to and except the rapid unloading. The method aims to be a helpful supplement to assist in predicting failure loads and modes during experimental testing of columns in intermediate slenderness ranges. The method combines the iterative Newmark's approach for eccentrically loaded columns with the arc-length displacement control scheme to direct the load increments. The results obtained are verified with known behaviour of column buckling with respect to slenderness, mode of failures, imperfections etc. The method simulated the load-deflection and failure mode of columns of various slenderness with reasonable accuracy.
\end{abstract}

Keywords: Inelastic buckling, Newmark's method, Arc-length method

\section{Introduction}

A buckled member or column loses stiffness and behaves according to a reduced modulus that defines the stress-strain relation in the buckled state. But a buckled member still carries some load, though much lesser because of a reduced modulus. Factors that influence the buckling load of individual members are the Young's modulus, end boundary conditions, residual stresses and imperfections. These affect both the ultimate failure loads of the column and the mode of failure i.e. squashing or buckling mode. Since in real experimental tests ideal conditions do not exist a method to predict the failure loads and modes would be of valuable assistance to the researcher.

In the buckled state, the lateral deflection induces secondary moments on the member. This secondary moment can cause full or partial yielding of the section of the members. In past research constitutive laws for different loading paths viz., loading, unloading, reloading are derived for the column when the section is fully or partially yielded. Members with fully yielded sections will have greatest reduction in stiffness and are the most critical. For this purpose two commonly used formulae are the tangent modulus and reduced modulus. While the former underestimates the column strength, the latter overestimates. However both methods oversimplify the behaviour by using just one modulus, though it is actually a combination of two modulus - Young's modulus before yielding starts in the cross-section of the column, and tangent modulus in the yielded portions of the cross-section. The method described in this study uses available expressions for consideration of partial yielding and includes them in an automatic incremental-iterative scheme to simulate the inelastic buckling of columns.

\section{Review of Literature}

Studies on members over the years lead to the development of the commonly used column curves by the structural stability research council (SSRC). Murtha-Smith and Magyar [1] divided the behaviour of cold formed steel struts into prebuckling, buckling and postbuckling parts including torsional-flexural behaviour. Expressions for the chordal shortening due to lateral displacement and stress using the secant formula are given. The authors aim was at arriving closed-form solutions. Several procedures for getting the buckling and postbuckling behaviour of tubular columns are described by Chen and Han [2]. These include both approximate closed form solutions and iterative procedures that use empirical forms of the moment-curvature-axial load $(M-P-\Phi)$ relationships that considers elastic, compression zone yielding and both zone yielding for tubular sections given by Saleeb [3]. The work by Atluri and Kondoh [4] describes the influence of the local instability i.e. member buckling on the overall global instability of truss type structures. The buckling of a strut in the elastic 
range is considered and the buckled geometry of truss member is used to derive the axial shortening due to buckling. The authors provide factors for reduction in axial stiffness and member force.

The inelastic post-buckling behaviour of tubular struts was studied in detail by Chan and Kitipornchai [5].The method accounts for the change in member geometry during deformation, the change in section properties by partial yielding of sections, and most importantly, the influence of permanent plastic strains. Pinned and fixed ended tubular struts are analysed for varying slenderness ratios and the results were found in reasonable comparison with experimental tests. Kitipornchai et al., [6] extended this work to struts of rectangular hollow sections. The author also derived equations using a curve fitting for the yield surfaces of monosymmetric sections such as channels, angles and T-sections. A 'lower bound theory' was used and numerical integration was carried out for the meshed cross-section to improve the accuracy of the results and curve fitting constants. From the literature it is evident that inelastic buckling of columns is mainly concentrated on closed form expressions and finite segment ([5], [6]) or finite element methods. While closed form expressions generally provide deflection estimates it is difficult to estimate the actual critical loads or mode of failure. FEM using commercial software requires careful application and selection of model parameters and element types. Ghazijahani et al [7] conducted experiments to evaluate the inelastic buckling capacity of short columns with large local imperfections or dents. Further, Bai and Jin [8] propose an empirical formula to approximate the critical buckling strain of tubular columns in terms of the ratios of bending strain to axial strain and the ratio of wall thickness to radius.

\section{Methodology}

Since by assuming a perfect column for the member it is not possible to get deflection estimates of a buckled member, a member with slight imperfection is assumed to trace an axial load-lateral deflection of the member. Therefore the deflection at the critical load (which is less sudden and well defined as compared to an ideal column) can be estimated. This becomes similar to a beam-column since the lateral deflection due to the axial load is caused only by the moments caused by the secondary effects. Moment-curvature is the defining relationship for tracing the member buckling behaviour. The core of the procedure used in this study is the Newmark's method [2] which use the $M-P-\Phi$ relations derived by Saleeb [3] particularly for tubular members. The assumptions taken for this method are: (1) the plane section remains plane after deformation and (2) shear deformation is neglected. The member with its deflected shape and loading is shown in Fig. 1. The $M-P-\Phi$ curve and the cross-section stress distribution at the defining points are shown in Fig. 2 and 3 respectively.
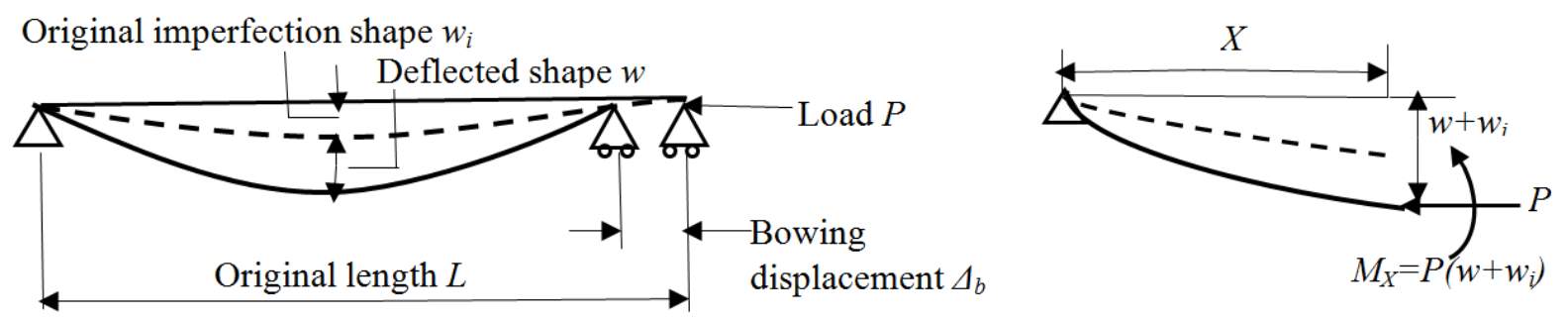

Fig. 1: Buckling in the imperfect column.

In the Fig. $3 m, p$ and $\varphi$ are nondimensionalised quantities of the moment, axial load and curvature with respect to the values at the start of initial yielding. $m_{p c}$ is $m$ when the cross-section is fully yielded.

$$
m=M / M_{y} ; \varphi=\Phi / \Phi_{y} ; p=P / P_{y}
$$

The nonlinear moment-curvature in Fig. 3 may be expressed for a given axial load by the piecewise relationships.

$$
m=\left\{\begin{array}{c}
a \varphi \quad\left(\varphi \leq \varphi_{1} ; \text { elastic }\right) \\
b-c / \sqrt{\varphi} \quad\left(\varphi_{1}<\varphi \leq \varphi_{2} ; \text { yielded in compression }\right) \\
m_{p c}-f / \varphi^{2} \quad\left(\varphi_{2}<\varphi ; \text { yielded both sides }\right)
\end{array}\right.
$$




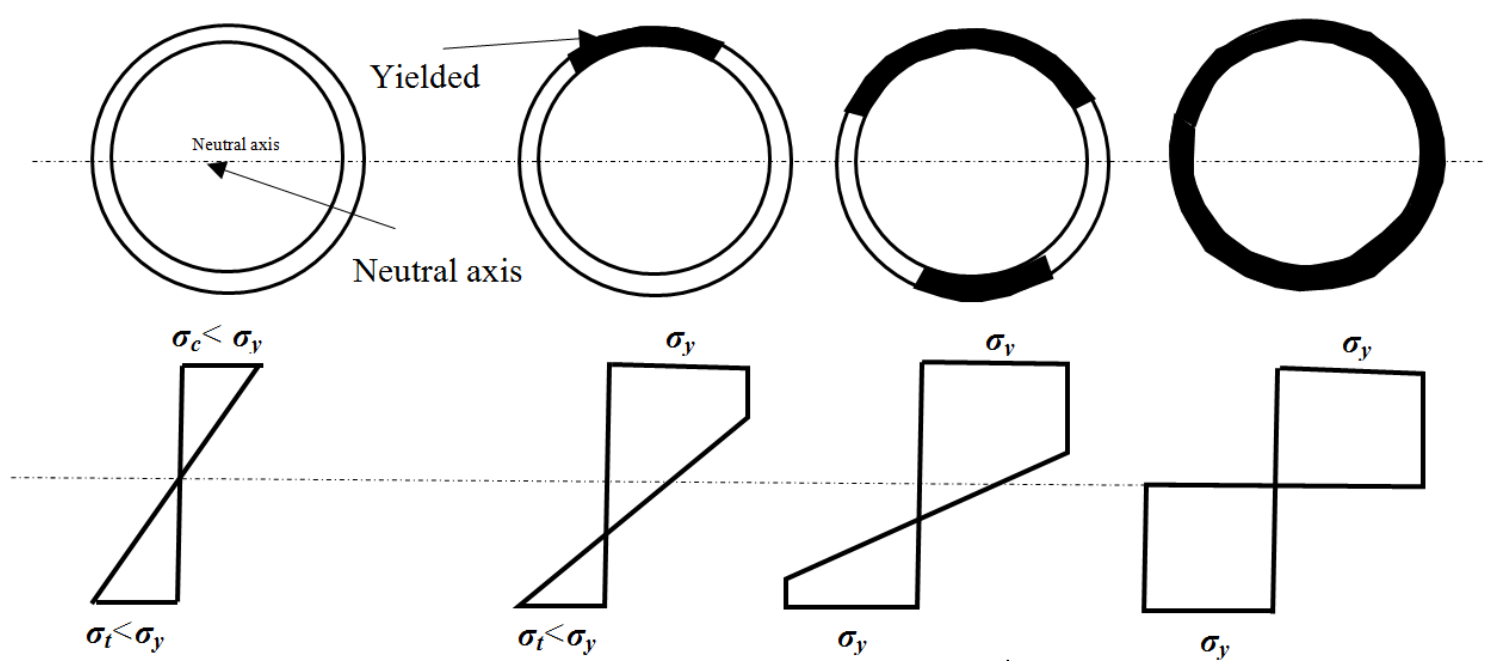

Fig. 2: Cross-section stress distribution.

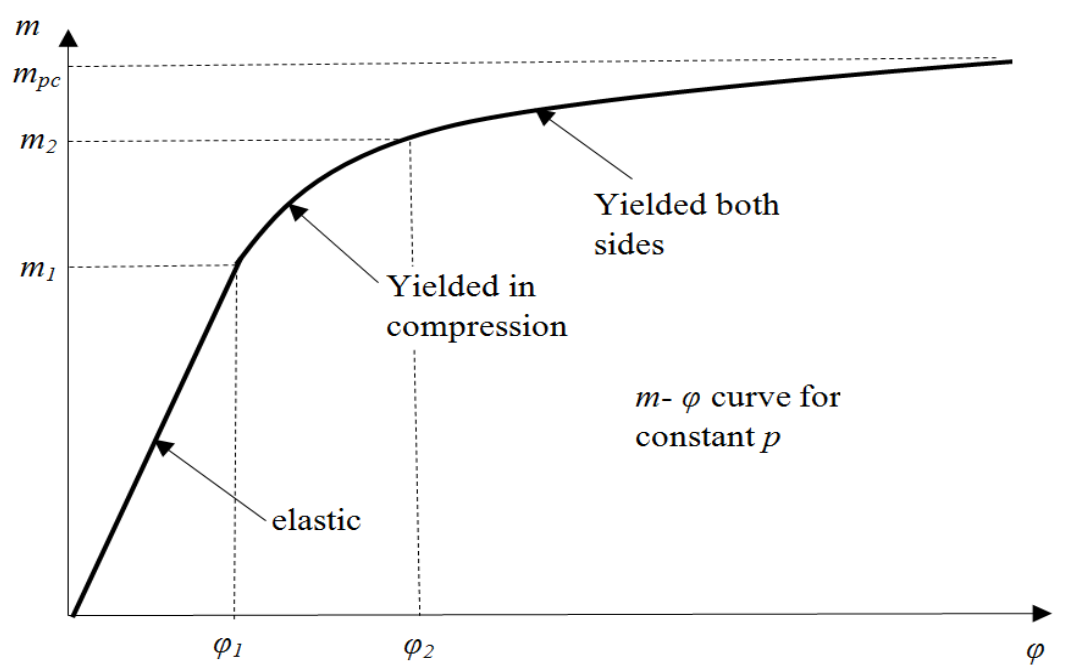

Fig. 3: Moment-curvature-load relationship of imperfect tubular column [3].

The unknowns $a, b, c$ and $f$ were obtained in reference [2] from curve fitting to get approximate expressions for them. The limiting values of non-dimensional moment and curvature in Fig. 3 when section is elastic, yielded in compression and yielded both sides are given as:

$$
\begin{gathered}
m_{1}=\varphi_{1}=1-p \\
m_{2}=\left\{\begin{array}{c}
1+0.21 p-1.05 p^{2} \text { for }(0 \leq p \leq 0.4) \\
1.528(1-p) \text { for }(0.4<p \leq 1)
\end{array}\right. \\
\varphi_{2}=\left\{\begin{array}{c}
1 /\left(1-1.395 p+1.206 p^{2}\right) \text { for }(0 \leq p \leq 0.4) \\
2.625(1-p) \text { for }(0.4<p \leq 1)
\end{array}\right. \\
m_{p c}=\left\{\begin{array}{c}
1.273\left(1-1.18 p^{2}\right) \text { for }(0 \leq p \leq 0.65) \\
1.82(1-p) \text { for }(0.65<p \leq 1)
\end{array}\right.
\end{gathered}
$$

The Newmark's method computes deflections at various stages of loading inside arc-length method which controls the load increments to trace a reasonable load-deflection path of buckling members. The method employs the relations provided above. The details of the arc-length method which can be found in standard nonlinear analysis text books (Reddy [9] and Crisfield [10]) are not shown in this paper. 


\subsection{Newmark's numerical procedure}

This method considers the equilibrium at each segment including the bending moments due to secondary effects that a member has been divided into. The procedure requires two levels of iterations: one in Newmark's method for deflections to converge and one for convergence in the arc length iterations. When interested only in the midspan deflection it is enough to divide only into 2 segments. Writing with actual moments (dimensionalised). The steps are given below

$$
M_{\text {internal }}=M_{\text {external }}+P w
$$

i. The initial imperfection in form of sinusoidal function gives maximum displacement at midspan. In absence of external lateral loads the moment is purely from axial load $M=P w=P \delta_{c}$ where $\delta_{c}$ as transverse deflection $w$ at midspan

ii. The $M-P-\Phi$ given earlier in terms of the nondimensionalised quantities is used in finding the appropriate curvature. Then the moment and curvatures are converted back to their dimensionalised values by multiplying with $M_{y}$ and $\Phi_{y}=M_{y} / E I$ where $E$ is the Young's modulus and $I$ is the moment of inertia of the cross-section.

iii. If the curvature in each segment is taken as quadratic the contributions to the slope from each segment can be calculated. For only two segments the contribution to the slope due to curvature at the midspan and at ends takes the form

$$
\kappa=h\left(\varphi_{p}+10 \varphi_{L / 2}+\varphi_{L}\right) / 12
$$

Where $h$ is the length of a segment, which in this case is $L / 2$. But since there are no moments at the ends (since $w$ is zero at ends) there is no calculated value of curvature at the ends and the above formula simplifies to

$$
\kappa=\left(5 L \varphi_{L / 2}\right) / 12
$$

iv. The slope is computed from all the contributions of curvature

$$
\theta=\kappa / 2
$$

v. The new lateral deflection $w_{a}$ is computed from slope at midspan along the length of the segment $h$

$$
w_{a}=h \theta
$$

vi. Convergence is checked between new lateral deflections $w_{a}$ and previous ones $w_{o}$ and steps (i) to (v) are repeated till satisfied

$$
\operatorname{conv}=\left|w_{a}-w_{o}\right| / w_{a}
$$

The procedure is said to have diverged when conv exceeds a high value, say 50. Convergence is also said to have failed when number of iterations is high, say 10.

This Newmarks procedure requires a control scheme to trace the path till the ultimate load for which the arc-length method is used in this study.

\subsection{Insertion in the arc-length method}

First iteration of first arc-length increment:

The starting stiffness of the member is the linear axial stiffness $\boldsymbol{K}^{\prime}=E A / L$. Assume initial values of load factor $\lambda$ and displacement increments and initial displacement. Note that axial shortening displacements are given by $u$ and lateral displacements by $w$. The reference load $P_{\text {ref }}$ is taken as a fraction $n$ of the minimum of the Euler critical load $P_{\text {Euler }}$ and squash load $P_{y}$. 


$$
\begin{aligned}
& \Delta \lambda_{1}^{0}=1 ; \Delta u=0 ; u_{0}=0 \\
& P_{\text {ref }}=\min \left(P_{\text {Euler }}, P_{y}\right) / n
\end{aligned}
$$

The Newmark's procedure calculates new value of the lateral displacements. The axial stiffness is then modified assuming the quadratic relationship between lateral displacement and the shortening due to the bowing effect (Jayachandran et al [11]). The member axial force is given by $N$ and the mid span lateral deflection by $\delta_{c}$. The converged value of lateral displacement $w_{a}$ from the Newmarks method is used as $\delta_{c}$.

$$
\begin{aligned}
& \boldsymbol{K}^{\prime}=\frac{1}{\left[\left(\frac{1}{\frac{A}{L}(E)}\right)+\frac{F_{\Delta} L}{N}\right]} \\
& F_{\Delta}=\left[1-\frac{1}{1+\frac{2}{3}\left(\frac{\delta_{c}}{L}\right)^{2}}\right]
\end{aligned}
$$

As per the arc-length procedure the imbalance force using this new $\boldsymbol{K}^{\prime}$ and the displacement iterative changes from the reference load and imbalance force are found and added to initial displacement. The arc-length change is computed from this displacement increments.

First iteration of other arc-length increment:

For the new load level $\lambda$, the Newmark's method is used to find the new $\delta_{c}$ for calculating the new $\boldsymbol{K}^{\prime}$. The previous converged $u$ is set as the starting value for this increment and the increment in arc length and load factor are found from the concerned formulas. The iterative change in displacement due to reference load is fixed for a whole arc-length increment. Only the iterative change due to the imbalance force found from the new $\boldsymbol{K}^{\prime}$ changes for each iteration. The total iterative change in displacement is added to the previous displacement and convergence is checked. A new iteration starts if it is not satisfied.

Non-first iterations:

For non-first iterations the Newmark's procedure is not performed and the same $\boldsymbol{K}^{\prime}$ as starting iteration. Therefore this can be thought of as a modified arc-length scheme (where stiffness modification occurs only in starting iterations of a load increment). The two parts of iterative displacement changes (due to reference load and due to imbalance force) are found and the quadratic equation (refer [9] or [10]) involving these iterative displacement changes are solved and the root chosen to get the correct value of iterative change in load factor $\delta \lambda$. The iterative change in load factor and displacements is added to the incremental changes found from previous iterations and convergence checked. The iterations are stopped when the load reached is very close to the maximum load i.e $n$ times $P_{r e f}$. This is taken as the ultimate load $P_{u}$. Even in cases when there is divergence or no convergence in the Newmark's method (due to excessive deflections without cross section yielding in very slender members) the load reached is taken as $P_{u}$.

The Newmarks-arc-length procedure was initially studied for single columns with few different degrees of imperfection and slenderness ratios and were checked in comparison to known column behaviours.

\section{Results}

A member was analysed for the effect of the imperfection $w_{i}$ for a fixed slenderness ratio with the same cross-section, length and properties as that given for the example in [2], namely: Depth of tubular section $=4.5$ in, wall thickness $=0.9375$ in, length of member $=125$ in, elastic modulus $=30,000 \mathrm{ksi}$, yield stress $=36 \mathrm{ksi}$. The results are plotted in Fig. 4 . Note that the axial load and displacement are normalised with respect to the yield axial load and displacement respectively. Next for a fixed degree of initial imperfection $\delta_{c}=0.001 \mathrm{~L}$ the slenderness ratios were varied by varying the depth of the various tubular sections. The other properties were: wall thickness $=4 \mathrm{~mm}$, length of member $=1000 \mathrm{~mm}$, elastic modulus $=2 \times 10^{5}$ $\mathrm{N} / \mathrm{mm}^{2}$, yield stress $=250 \mathrm{~N} / \mathrm{mm}^{2}$. The plot is shown Fig. 5 with properties in Table 1. 
In Fig. 6 the slenderness of the members analysed in Fig. 5 are plotted against the ratio of their ultimate strength to the ultimate strength of ideal columns $\left(\mathrm{P}_{\text {ideal }}\right)$ of the same dimensions. This is plotted to highlight the capability of the scheme to differentiate the two different modes of failures namely axial yielding (squashing) and buckling which happen in the short and slender extremes of slenderness ratio. The middle vertical line is the limiting slenderness ratio $2 \pi \sqrt{E / f_{y}}$ for the given material properties.

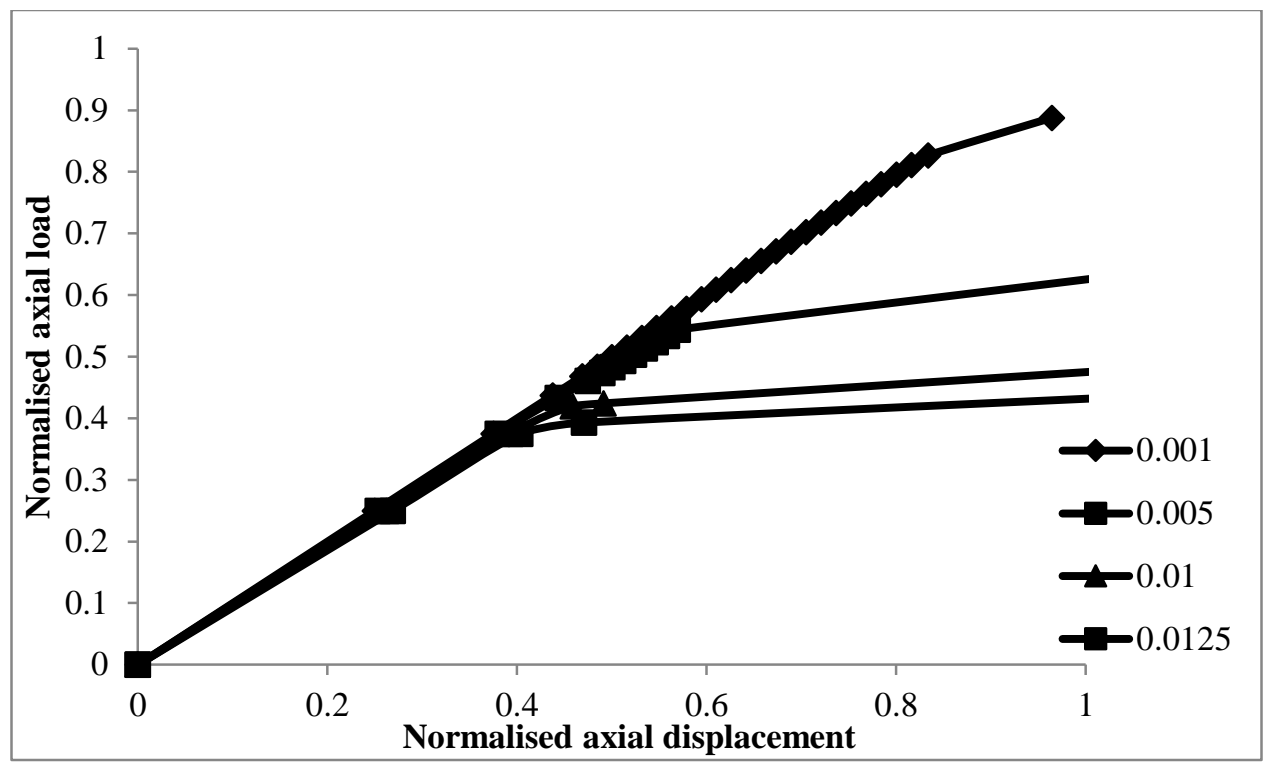

Fig. 4: Load vs axial shortening with varying imperfection.

As expected the shortening increases faster with degree of imperfection. Also expected trends of decreasing ultimate critical load $P_{u}$ with increasing slenderness is seen. It is seen that for very less slenderness of 54, the actual slenderness is lesser than the limiting slenderness and thus compression yielding $m_{1}$ is not reached. This is because the actual moment is much lesser than yield moment $M_{y}$ due to large value of $I$. This column can be considered to lie in the short range of slenderness and fail by squashing. The procedure is stopped because maximum load is reached. On the contrary, with high slenderness compared to limiting slenderness (slender column) $m_{1}$ is not reached because of the high curvature which means a high lateral deflection $w$. The column buckles elastically without even reaching the compression yielding. The procedure is stopped because of excessive deflection (divergence). Thus the procedure is suitable for both extremes of slenderness and the only difference is in the way it is judged.

Also from Fig. 6 it is seen that maximum reductions in ultimate loads compared to ideal columns of same dimension occurs in intermediate slenderness columns close to the limiting slenderness. This is in agreement with experimental observations from tests conducted by the SSRC [12].

Table 1: Properties of sections with initial imperfection $1 \mathrm{~mm}$.

\begin{tabular}{|c|c|c|c|c|}
\hline Slenderness ratio & Depth of section & $\begin{array}{c}\mathrm{P}_{\text {ideal }}=\min \left(\mathrm{P}_{\text {Euler }},\right. \\
\left.\mathrm{P}_{\mathrm{y}}\right)\end{array}$ & $\mathrm{P}_{\mathrm{u}}$ & $\mathrm{P}_{\mathrm{u}} / \mathrm{P}_{\text {ideal }}$ \\
\hline 54 & $50 \mathrm{~mm}$ & $144500 \mathrm{~N}$ & $136747 \mathrm{~N}$ & 0.946 \\
\hline 76 & $35 \mathrm{~mm}$ & $97500 \mathrm{~N}$ & $83096 \mathrm{~N}$ & 0.852 \\
\hline 96 & $27 \mathrm{~mm}$ & $61037 \mathrm{~N}$ & $51634 \mathrm{~N}$ & 0.846 \\
\hline 103 & $25 \mathrm{~mm}$ & $48453 \mathrm{~N}$ & $40753 \mathrm{~N}$ & 0.841 \\
\hline 127 & $20 \mathrm{~mm}$ & $24808 \mathrm{~N}$ & $21553 \mathrm{~N}$ & 0.869 \\
\hline
\end{tabular}




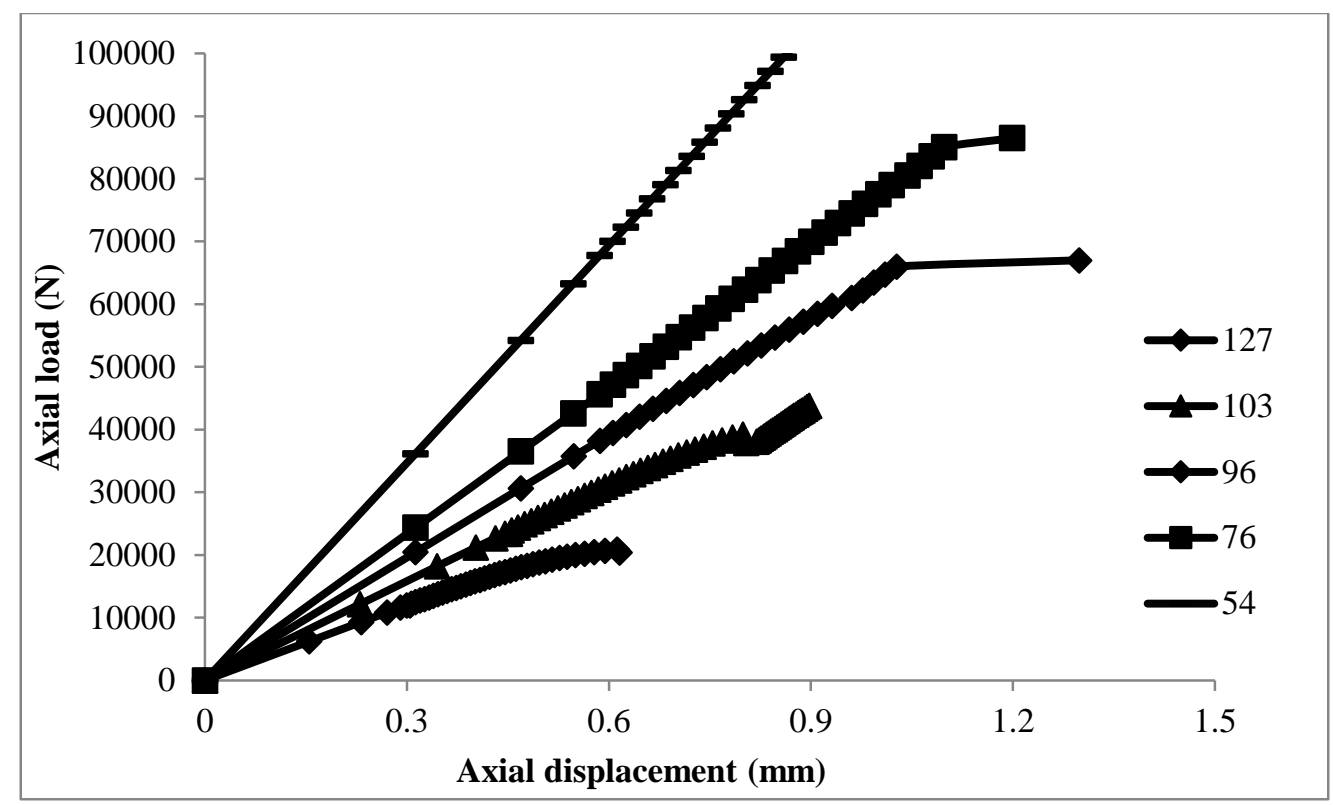

Fig. 5: Load vs axial shortening with varying slenderness ratios.

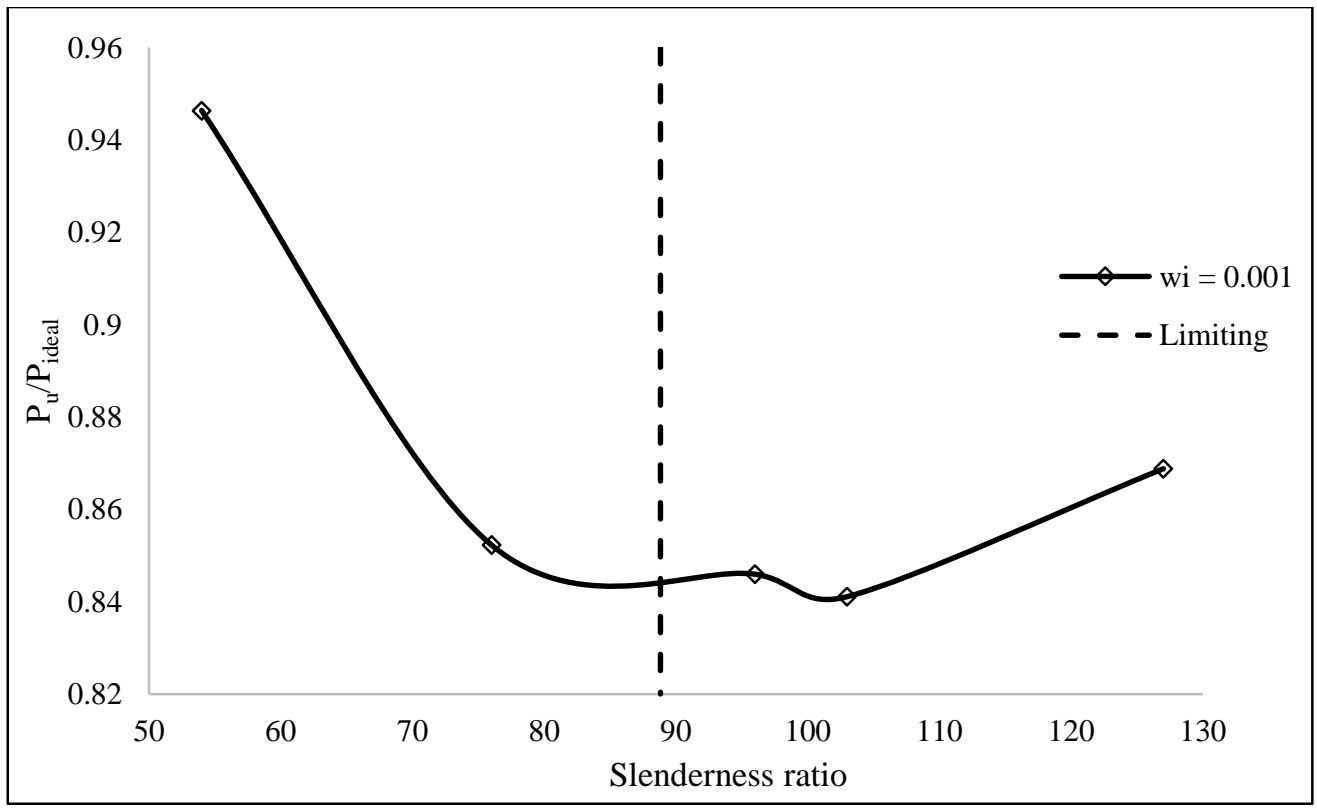

Fig. 6: Ultimate load reductions with slenderness ratio variation.

\section{Conclusions}

The study replicates analytically the inelastic buckling of columns which through a fully automatic incrementaliterative scheme. The study addresses the limitations in predictions of the closed form expressions and modelling complexities of FEM. The Newmark's method for imperfect columns is used for calculating the lateral displacement and modifying the axial stiffness. Arc-length control is used to direct the load increments and arrive at the ultimate loads. The method was able to identify both the squash and buckling mode of failures in columns of various slendernesses. Thus the method is a particularly useful tool to pre-judge the ultimate loads and failure modes of intermediate slenderness columns which show tendency to fail by either of the modes during experimental testing. 


\section{References}

[1] E. Murtha-Smith and P. Magyar, "Cold-formed steel channel struts," J. Struct. Eng., vol. 117, no. 4, pp. 1276-1293, 1991.

[2] W. F. Chen and D. J. Han, Tubular members in offshore structures. Pitman Publishing Ltd, 1985.

[3] A. F. Saleeb, "Near-bottom bend of flowlines," M.S. thesis, School. Civil. Eng., Purdue Univ., West Lafayette, IN.

[4] K. Kondoh and S. N. Atluri, "Influence of local buckling on global instability: Simplified, large deformation postbuckling analysis of plane trusses," Comput .Struct., vol. 21, no. 4, pp. 613-627, 1985

[5] S. L. Chan and S. Kitipornchai, "Inelastic post-buckling behaviour of tubular struts," J. Struct. Eng., vol. 114, no. 5, pp. 1091-1105, 1988.

[6] S. Kitipornchai, F. G. A. Al-Bermani, and S. L. Chan, "Geometric and material nonlinear analysis of structures comprising rectangular hollow sections," Eng. Struct.., vol. 10, pp. 13-23, 1988.

[7] T. G. Ghazijahani, H. Jiao, and D. Holloway, "Plastic buckling of dented steel circular tubes under axial compression: An experimental study," Thin Walled Struct., vol. 92, pp. 48-54, 2015.

[8] Y. Bai and W. L. Jin, Marine structural design. Butterworth-Heinemann, 2016.

[9] J. N. Reddy, An introduction to nonlinear finite element analysis. OUP Oxford, 2004.

[10] M. A. Crisfield, Nonlinear finite element analysis of solids and structures: Essentials: 1. Wiley-Blackwell, vol. 1, 1996.

[11] S. A. Jayachandran, V. Kalyanaraman, and R. Narayanan, "A co-rotation based secant matrix procedure for elastic postbuckling analysis of truss structures," Int. J. Struct. Stab. Dyn. , vol. 4, no. 1, pp. 1-19, 2004.

[12] E. H. Gaylord, C. N. Gaylord, and J. E. Stallmeyer, Design of steel structures. New Delhi: McGraw-Hill, 2010. 